\title{
Clinical association of progesterone receptor isoform A with breast cancer metastasis consistent with its unique mechanistic role in preclinical models
}

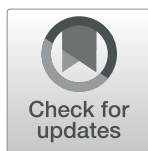

Rayna Rosati, Kailey Oppat, Yanfang Huang, Seongho Kim and Manohar Ratnam * (D)

\begin{abstract}
Background: Luminal breast cancer (L-BCa) comprises the majority of incurable, distally metastatic breast cancer cases. Estrogen supports growth of L-BCa cells but suppresses invasiveness. Estrogen also induces the progesterone receptor (PR). Invasiveness and metastasis of L-BCa cells is supported by the short PR isoform (PR-A), in response to the range of pre- and post-menopausal plasma hormone levels, by counteracting the effects of estrogen via micro RNA-mediated cross-talk with the estrogen receptor (ER). PR-B directly supports L-BCa invasion and metastasis and also inhibits tumor growth, both only at high progesterone levels. As public datasets on L-BCa tumors cannot distinguish PR-A, this study was designed to seek clinical evidence for the role of PR-A in metastasis in comparison with PR-B and ER.
\end{abstract}

Methods: Measurement of tumor PR-A, PR-B and ER mRNA expression in 125 treatment-naive primary L-BCa patients with differential node involvement and analysis using linear mixed effects models. Transcriptional activity assays of PR-A and PR-B.

Results: Lymph node involvement was strongly associated with PR-A expression (median, 3-fold higher vs. nodenegative), independent of age, pathologic type, tumor grade, HER2 and PR-B. PR-B and ER correlated weakly with PR-A, but whereas PR-B and the PR-A/PR-B ratio were not significantly associated with node involvement, ER weakly negatively correlated with node positivity. PR-A was hypersensitive to mifepristone compared with PR-B.

Conclusions: Taken together with previous mechanistic studies, the findings provide clinical evidence in support of the role of PR-A in L-BCa metastasis. They also suggest the possibility of developing selective PR-A modulators for future interventions in appropriate clinical situations.

Keywords: Breast cancer, Progesterone receptor, Estrogen receptor, Estrogen, Progesterone, Mifepristone

\footnotetext{
* Correspondence: ratnamm@karmanos.org

Barbara Ann Karmanos Cancer Institute and Department of Oncology, Wayne State University School of Medicine, 4100 John R, HWCRC 840.1, Detroit, MI 48201-2013, USA
}

(c) The Author(s). 2020 Open Access This article is licensed under a Creative Commons Attribution 4.0 International License, which permits use, sharing, adaptation, distribution and reproduction in any medium or format, as long as you give appropriate credit to the original author(s) and the source, provide a link to the Creative Commons licence, and indicate if changes were made. The images or other third party material in this article are included in the article's Creative Commons licence, unless indicated otherwise in a credit line to the material. If material is not included in the article's Creative Commons licence and your intended use is not permitted by statutory regulation or exceeds the permitted use, you will need to obtain permission directly from the copyright holder. To view a copy of this licence, visit http://creativecommons.org/licenses/by/4.0/ The Creative Commons Public Domain Dedication waiver (http://creativecommons.org/publicdomain/zero/1.0/) applies to the data made available in this article, unless otherwise stated in a credit line to the data. 


\section{Background}

Breast oncogenesis may occur over decades. The majority $(>78 \%)$ of initial breast cancer diagnoses are in women over 50 years of age [1] and median age, 61 years [2]. The majority of breast cancer (BCa) cases are of the luminal subtype (L-BCa), which is estrogen receptor (ER)-positive [3] and continues to express ER into advanced stages [4]. Primary L-BCa is exquisitely sensitive to anti-estrogens. Unfortunately, $\mathrm{L}-\mathrm{BCa}$ has frequently metastasized prior to diagnosis and the metastatic tumors may also appear long after cessation of therapy [5, 6]. At least $20 \%$ of $\mathrm{BCa}$ patients have $\mathrm{ER}+$ tumors showing distal metastasis [7]. Metastatic L-BCa is typically incurable. Our previous studies have mechanistically linked invasiveness and metastasis of L-BCa to a novel hormonal mechanism $[8,9]$.

Growth of ER+ breast tumors is supported by estrogen but the hormone suppresses tumor invasiveness, regardless of hormone sensitivity of growth and suppresses tumor progression [10-16]. In contrast, progestins support invasiveness and metastasis in L-BCa cells $[17,18]$. A single gene encodes two isoforms of the progesterone receptor (PR), PR-A and $\mathrm{PR}-\mathrm{B}$, via alternative promoter usage. PR-B only differs from PR-A in having an aminoterminal segment of 164 residues containing the activation function, AF3 [19]. Despite overlap in the gene regulatory patterns of $\mathrm{PR}-\mathrm{B}$ and $\mathrm{PR}-\mathrm{A}$, the two isoforms also show a clear distinction in terms of the strength of this regulation and also in the target genes that they activate or repress [19-21]. The two PR isoforms can heterodimerize and regulate a small set of unique genes [20, 22]. PR-A and PR-B show similar levels of expression in normal breast but breast oncogenesis is associated with loss of this coordinate expression, mostly resulting in a high PR-A:PR-B ratio, in early as well as progressed lesions [23]. The higher ratio of PR-A to PR-B is generally due to PR-A overexpression and has been reported to be negatively associated with disease free survival (DFS), although the mechanistic underpinnings were not established [24].

The pre-menopause plasma estrogen level is $1.4 \mathrm{nM}-$ $1.6 \mathrm{nM}$ (follicular phase), and $3.6 \mathrm{nM}-4.2 \mathrm{nM}$ (luteal phase) [25]. Progesterone levels in the plasma range from $<4 \mathrm{nM}$ in the follicular phase, up to $>50 \mathrm{nM}$ in the luteal phase [26]. After menopause, the circulating hormone levels show a marked decline (median value for estrogen of $0.14 \mathrm{nM}$ and median value for progesterone of $0.13 \mathrm{nM}$ ), but hormone levels of up to $\sim 1 \mathrm{nM}$ may be retained in breast tissue $[27,28]$. Previous in vitro studies in the literature had suggested progesterone induces invasiveness of $\mathrm{BCa}$ cells principally via PR-B $[29,30]$ although clinical observations noted more frequent elevation of PR-A in ductal carcinoma in situ (DCIS) and invasive breast lesions [23] and associated PR-A rather than PR-B with lower DFS [24]. Notably, those in vitro studies were conducted using relatively high progesterone concentrations (corresponding to luteal stage, pregnancy or pharmacological plasma levels) and excluded estrogen signaling [29-33].

We have reported studies covering the full ranges of pre-menopausal and post-menopausal levels of estrogen and progesterone, conducted with both estrogen and progesterone signaling present, using T47D, ZR-75-1 and BT474 cells as well as isogenic recombinant T47D cells expressing a single PR isoform [8]. Previous studies of the effects of high dose progesterone on invasion and metastasis utilized models in which estrogen signaling was absent. Therefore we took into account the possibility that cross-talk with estrogen signaling may influence regulation of invasiveness by progestins at physiological hormone levels. At concentrations $<0.01 \mathrm{nM}$, estrogen strongly suppressed invasiveness of ER+ BCa cells. At relatively low concentrations $(<1 \mathrm{nM})$, progestins abrogated inhibition of invasiveness caused by estrogen. It took relatively higher concentrations of progestins, (in the range of $5 \mathrm{nM}-50 \mathrm{nM}$ ) to induce invasiveness in the absence of estrogen, in a progestin dose-dependent manner. It was the PR-A isoform that mediated rescue of invasiveness from estrogen regulation by progesterone and this effect was uninfluenced by PR-B [8]. In contrast, estrogen-independent induction of invasiveness by progestin was mediated by PR-B and this occurred at either pregnancy-associated or pharmacological levels of progestin [8]. When PR-A was 2-3 fold overexpressed in PR-A+/PR-B+ cells, a lower concentration of progestin $(<0.2 \mathrm{nM})$ completely rescued invasiveness. These hormonal effects on invasiveness were independent of HER2 status. Thus progesterone supports BCa cell invasiveness by counteracting estrogen, acting exclusively through PR-A, at hormone concentrations corresponding to the full physiological ranges of estrogen and progesterone levels, both prior to and after menopause [8].

To interrogate the role of the ER-PR-A crosstalk in metastasis, we investigated selective crosstalk mechanisms of PR-A with ER via microRNAs (miRNAs), which have an extensive role in estrogen signaling [9]. Among miRNAs regulated by estrogen or progesterone, miR92a-3p and miR-26b-5p mediated the PR-A-ER crosstalk. Progesterone plus PR-A suppressed induction of miR-92a-3p and repression miR-26b-5p by estrogen. This resulted in regulation of genes involved in invasiveness and metastasis and also completely rescued invasiveness in vitro. In murine xenograft models, when miR-92a-3p was constitutively expressed or when miR26b-5p was inhibited, metastasis was profoundly suppressed, similar to knockdown of PR [9]. Notably, these two miRNAs did not regulate each other and, based on quantitative changes in mRNAs of genes involved in 
invasion and/or metastasis using a commercially available pathway cDNA array, we found that the two micro RNAs mediate independent hormone-regulated pathways controlling invasion and metastasis that are convergent. Therefore, the strong suppression of invasiveness by even very low concentrations of estrogen $(\sim 0.01 \mathrm{nM})$ may be the net effect of sub-optimal regulation of these two miRNAs by estrogen. In primary tumors, PR-A expression showed negative correlation with miR-92a-3p and positive correlation with miR-26b-5p, but the strength of this correlation was moderate, as expected because of variable stromal content in resected tumor specimens, the likely influence of variability in the range of plasma progesterone levels in postmenopausal women and differences in expression of a compliment of coregulators and downstream effectors. The mechanistic studies suggest that hormonal crosstalk of PR-A with ER enables metastasis of luminal $\mathrm{BCa}$, notably by opposing regulation of critical microRNAs by estrogen. This occurs even at hormone levels corresponding to plasma hormone status in the follicular stage and post-menopause.

Public RNAseq databases on the transcriptome profiles of breast tumors, such as The Cancer Genome Atlas (TCGA), do not distinguish PR-A, because it has multiple transcripts arising from alternative promoter usage that only differ from the PR-B transcript with respect to the positions of their $5^{\prime}$ ends. Therefore, in this study, we undertook a prospective analysis to clinically validate the role of PR-A in metastasis. We further investigated the possibility of isoform-selective modulation of PR-A.

As described above, previous studies have rigorously elucidated a mechanistic model of breast cancer metastasis that should predict the manner in which expression of ER, PR-A and PR-B are associated with L-BCa metastasis (Fig. 1). In this model, PR-A is induced by estrogen and thus ER limits its own ability to suppress invasion and metastasis. Additionally, PR-A may also be further up-regulated in $\mathrm{L}-\mathrm{BCa}$ via mechanisms that are independent of estrogen. PR-B on the other hand requires relatively high physiological levels of progesterone

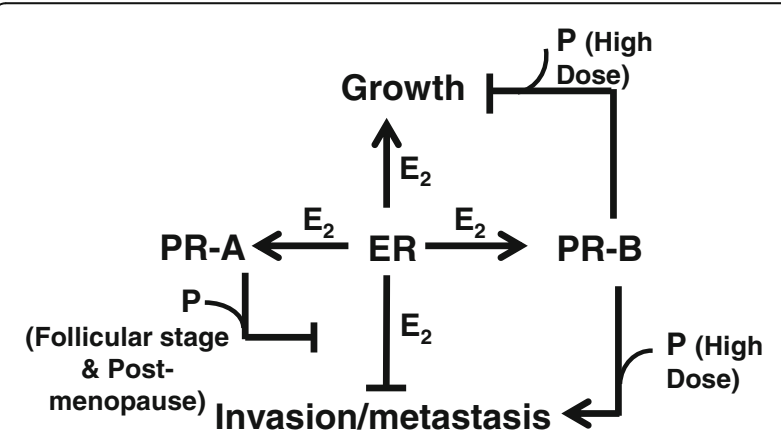

Fig. 1 Schematic showing cross-talk between estrogen (E2) and progesterone $(\mathrm{P})$ signaling in regulating progression of breast cancer (premenopausal luteal stage and pregnancy associated levels) to induce metastasis. Therefore, the model predicts a strong clinical association of PR-A with metastasis of primary L-BCa but, at best, a weak association of PR-B with metastasis. The model also predicts that the association of PR-A with metastasis should be uninfluenced by the relative expression of PR-B as PR-B influences metastasis by a separate mechanism and only at high progesterone levels. At the same time the model predicts a likely negative association of ER with metastasis. The mechanistic studies also predict that expression of HER2 will not affect the association of PR-A with metastasis.

\section{Methods}

Cell line models, tissue specimens and reagents

Isogenic T47D-A (ER+/PR-A+/PR-B-null) and T47D-B (ER+/PR-B+/PR-A-null) recombinant cells were a generous gift from Dr. Katherine Horowitz (University of Colorado, Denver, CO) and were cultured as described previously All human tumor samples, from female patients, classified as treatment naïve $\mathrm{ER}+/ \mathrm{PR}+$ primary breast carcinoma were obtained from the Cooperative Human Tissue Network (CHTN). For each tumor tissue selected, the largest dimension was in the range of $1.5 \mathrm{~cm}-2 \mathrm{~cm}$. Lipofectamine 2000 was from Thermo Scientific (product number 78410). R5020, and RU486 (mifepristone) were purchased from Sigma Aldrich (Saint Louis,MO).

\section{Isolation and measurement of mRNA}

Total RNA was isolated from tissues using the Purelink RNA Mini Kit (ThermoFisher Scientific, Grand Island, NY). Breast tumor tissue lysates were prepared by suspending $30 \mathrm{mg}$ of tissue in $500 \mu \mathrm{l}$ of the lysis buffer and homogenized using the PRO200 tissue homogenizer (catalog no. 01-01200, BioGen) for 15 s on ice. Homogenized solution was centrifuged at high, 15,000 $\mathrm{x}$ g, and supernatant was used for RNA extraction. Reversetranscription PCRs were performed using high-capacity complementary DNA archive kit (Life Technologies, Inc., Carlsbad, CA). cDNA was measured by quantitative real-time RT-PCR using TaqMan probes and the StepOne Plus real-time PCR system (Life Technologies). All RNA measurements were performed in biological triplicates, and all $\mathrm{Ct}$ values were normalized to intrasample GAPDH (mRNA).

\section{Statistical methods}

A total of 125 breast cancer tumor tissues (extracted from 125 patients) were analyzed by real-time quantitative PCR (qPCR) to measure Ct values of Total PR, PR$A$ and PR-B. The qPCR was repeated 3 times for each RNA to generate biological replicates, resulting in 375 $\mathrm{Ct}$ values for each RNA. Note that the $\mathrm{Ct}$ value is defined as the number of cycles needed for the 
fluorescence to reach a specific threshold level of detection and is reciprocally correlated with the amount of template present in the reaction [34]. The efficiency of each primer pair $(E=2)$ was used to normalize the $\mathrm{qPCR}$ data and a relative gene expression value with regard to control was calculated using the equation $2^{\Delta C T_{\text {sample }}}$ $=2^{C t_{\text {control }}-C t_{\text {sample }}}$, where $C t_{\text {control }}$ is the $\mathrm{Ct}$ value for a housekeeping gene and $C t_{\text {sample }}$ is the $\mathrm{Ct}$ value for each amplification. The relative gene expression value of PRA was obtained by the equation $2^{\Delta C t_{\text {Total }} P R}-2^{\Delta C t_{P R-B}}$. In particular, if $\Delta C t_{\text {Total } P R}<\Delta C t_{P R-B}$, we assigned zero for the relative expression vale of PR-A [35]. To calculate the correlation coefficients in repeated measures [36] among RNAs, a linear mixed-effect modeling was used after each relative gene expression value was transformed with the Box-Cox transformation with two parameters due to zeros [37]. In addition, the associations between the lymph node status (positive vs. negative) and each of the expression levels of Total PR, PR-A and PR-B were examined using those 125 tumor tissues. In order to take into account three replicates in each measurement, a linear mixed-effects model was used to assess the associations. All statistical analyses were performed using the statistical software package $\mathrm{R}$ (https://www.rproject.org) and the Box-Cox transformation with two parameters were carried out using the function boxcoxfit available in the $\mathrm{R}$ package geoR.

\section{Other experimental methods}

Transient transfections and luciferase reporter assays have been described [38]. $\mathrm{IC}_{50}$ values for mifepristone were determined using GraphPad Prism dose-response analysis of the log-transformed drug concentrations and response.

\section{Results}

Prospective investigation of hormone receptor expression in relation to metastasis of L-BCa

The most reliable indicator of metastasis of a primary luminal breast tumor is nodal involvement, assessed primarily from axillary lymph node biopsies, especially sentinel node biopsies [39]. The inherent metastatic potential of a tumor, which is independent of treatment, determines the frequency and extent of tumor spread in the years spanning the time of occurrence of the oncogenic event and the initial cancer diagnosis as well as the years following cessation of treatment. Therefore, we used axillary lymph node status at time of diagnosis as an objective indicanaivtor of metastatic potential of the primary tumors to investigate whether PR-A expression in the tumors is associated with their ability to metastasize, independent of treatment.
We have previously reported that in ER+ primary $\mathrm{BCa}$ tumor specimens the relative protein levels of PR-A and PR-B are reflected by their relative mRNA levels [9]. However, public datasets on the transcriptome profiles of breast tumors, such as TCGA, do not distinguish PR-A from PR-B, because it has multiple transcripts arising from alternative promoter usage that only differ from the PR-B transcript with respect to the positions of their $5^{\prime}$ ends. Therefore, we prospectively examined treatmentnaïve primary ER+ breast tumors from 125 patients, using a standardized differential quantitative RT-PCR assay for PR-A and PR-B. The patient baseline characteristics are described in Table 1. They include similar numbers of patients with vs. without node involvement at time of diagnosis with mostly ductal carcinomas and lesions ranging from Grade I - Grade III. The tumors were predominantly HER2-negative. In these specimens, we examined expression of mRNAs for PR-A, PR-B and ER.

\section{Correlation of PR-A expression with those of total PR, PR- $B, E R$ and with node status}

PR-A expression correlated moderately with total PR ( $\mathrm{r}$ value, 0.66) and weakly with expression of PR-B ( $r$ value, 0.28 ) or ER ( $r$ value, 0.24 ) (Fig. 2a-c). In the unadjusted analysis, total PR (p, 0.028) and PR-A (p, 0.009) showed a significant (positive) association with node involvement with 1.7- and 2.9-fold higher median values vs. node negative tumors, respectively (Fig. 2d, e and Table 2). PR-B was not significantly correlated with node status and neither was the ratio of PR-A to PR-B (Fig. 2f, g and Table 2). In contrast, in the unadjusted analysis, ER notably showed a negative correlation with node involvement (p, 0.029) (Fig. 2h and Table 2).

When the data was adjusted by multivariable analysis for age, histology and grade, PR-A showed the strongest association with node positivity (t-value, 2.99; p, 0.003) (Table 2). Total PR showed a similar trend but neither PR-B nor the PR-A/PR-B ratio correlated significantly with node status. Further, adjusting to remove the effect of PR-B did not significantly affect the relationship between PR-A and node status in the univariable or multivariable analysis (Supplementary Table 1). The adjusted analysis weakened the negative correlation of ER with node involvement.

The ability of PR-A to predict metastasis held true when its expression level was dichotomized (low vs. high) by its median value, with OR $(95 \% \mathrm{CI})$ of 2.3 (1.53.5) $(p<0.001)$ in the multivariable logistic regression analysis that adjusted for age, histology and grade (Supplementary Table 2). Whereas total PR showed a similar trend, PR-B, the PR-A/PR-B ratio and ER did not predict metastasis (Supplementary Table 2). Moreover, adjusting to remove the effect of PR-B did not influence the 
Table 1 Patient baseline characteristics. The comparisons were conducted by Fisher's exact tests for categorical variables and by Kruskal-Wallis tests for continuous variables

\begin{tabular}{|c|c|c|c|c|}
\hline & \multicolumn{2}{|l|}{ Lymph node status } & \multirow[t]{2}{*}{ All $(\boldsymbol{N}=125)$} & \multirow[t]{2}{*}{$\mathrm{p}$} \\
\hline & Negative $(\boldsymbol{N}=62)$ & Positive $(\boldsymbol{N}=63)$ & & \\
\hline Age - median (range) & $59.5(25-87)$ & $54(35-87)$ & $57(25-87)$ & 0.054 \\
\hline Histologic type - no. (\%) & & & & 0.612 \\
\hline Ductal & $45(73)$ & $40(63)$ & $85(68)$ & \\
\hline Lobular & $9(15)$ & $15(24)$ & $24(19)$ & \\
\hline Mixed & $5(8)$ & $5(8)$ & $10(8)$ & \\
\hline None & $3(5)$ & $3(5)$ & $6(5)$ & \\
\hline Grade - no. (\%) & & & & 0.566 \\
\hline । & $10(16)$ & $7(11)$ & $17(14)$ & \\
\hline$\|$ & $27(44)$ & $26(41)$ & $53(42)$ & \\
\hline$\|$ & $24(39)$ & $30(48)$ & $54(43)$ & \\
\hline missing & $1(2)$ & $0(0)$ & $1(1)$ & \\
\hline HER2 status - no. (\%) & & & & 0.572 \\
\hline Negative & $46(74)$ & $50(79)$ & $96(77)$ & \\
\hline Equivocal & $9(15)$ & $5(8)$ & $14(11)$ & \\
\hline Positive & $3(5)$ & $2(3)$ & $5(4)$ & \\
\hline missing & $4(6)$ & $6(10)$ & $10(8)$ & \\
\hline
\end{tabular}

predictive ability of PR-A, either in the univariable or multivariable analysis (Supplementary Table 3).

Our study cohort had a limited number of HER2positive tumor specimens, reflecting the relatively low frequency of HER2 amplification in L-BCa. Specifically, the study included 96 HER2-negative specimens, 5 HER2-positive specimens, 14 specimens with equivocal HER2 status and 10 specimens in which data on HER2 expression was unavailable. Therefore, we repeated the univariable and multivariable analysis using specimens that were classified either as HER2-negative (96 specimens) or HER2-non-negative $(5+14=19$ specimens $)$. Including HER2 in this manner in the adjusted analysis did not alter the association of PR-A with node involvement (Supplementary Table 4). The house keeping gene used in all cases was GAPDH. The intra-sample $\mathrm{Ct}$ values for GAPDH were used to normalize for variabilities in the exact amount of RNA in each assay as well as possible variability in the efficiency of reverse transcription between samples or across experiments. We did not see an association between the raw GAPDH $\mathrm{Ct}$ values and node status or other tumor characteristics (Supplementary Figure 1).

\section{Selective antagonism of isoform A of PR}

As PR-A is likely functionally associated with L-BCa metastasis over a much broader range of physiological hormone status than PR-B, it was of interest to seek proofof-principle for whether it would be possible to selectively modulate the PR-A isoform as a potential future strategy for intervention in L-BCa metastasis. As studies have shown that administering low dose mifepristone for extended periods is well tolerated in humans and as our previous studies noted that a low $(1 \mathrm{nM})$ concentration of mifepristone only inhibited the effect of PR-A but not PR-B on invasiveness of L-BCa cells, we examined the relative sensitivities of the transcriptional activities of PR-A and PR-B to mifepristone. In a hormonedependent promoter activation assay using isogenic T47-D cells expressing either PR-A alone (T47D-A cells) or PR-B alone (T47D-B cells), PR-A was hyper-sensitive to mifepristone compared with $\mathrm{PR}-\mathrm{B}$, with $\mathrm{IC50}$ values for the drug of $0.45 \times 10^{-9} \mathrm{M}$ and $5.72 \times 10^{-9} \mathrm{M}$ respectively, for PR-A and PR-B (Fig. 3a). We then used the same isogenic cells to examine the mifepristone dosedependence for the two PR isoforms for inhibition of activation of HSD11B2, a known common endogenous gene target of PR-A and PR-B [39] Again, hormonedependent induction of the HSD11B2 mRNA by PR-A was highly sensitive to mifepristone, which was required at a much higher dose to inhibit PR-B (Fig. 3b).

\section{Discussion}

Diagnostic evaluation of PR status in primary breast cancer does not typically distinguish PR isoforms. However, two major clinical studies have suggested an association between PR-A and progression of L-BCa. In one study, coordinate expression of PR-A and PR-B observed in normal breast epithelial cells and in benign breast tumors was reportedly frequently disrupted in both DCIS 
A.

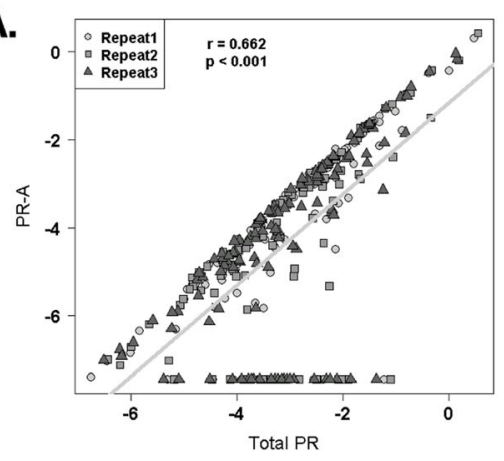

C.
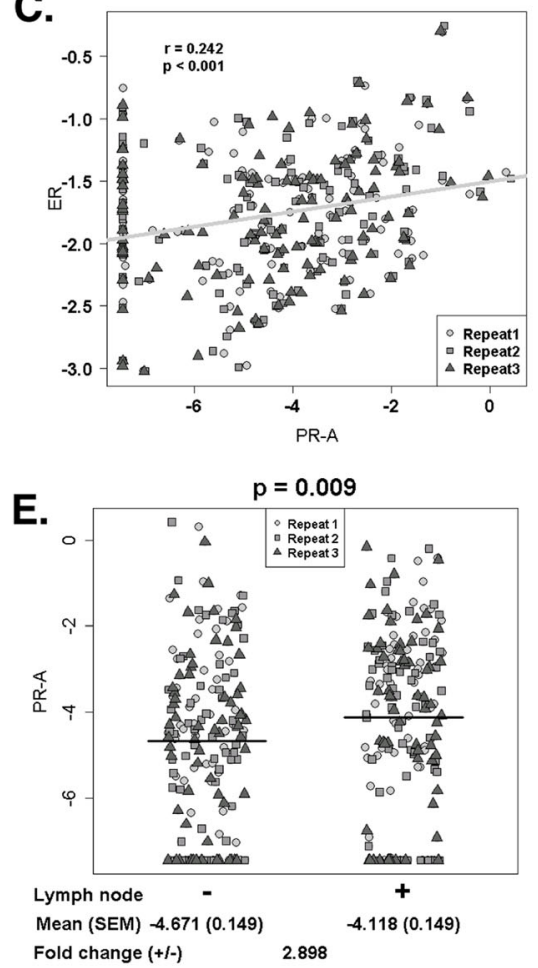

G.

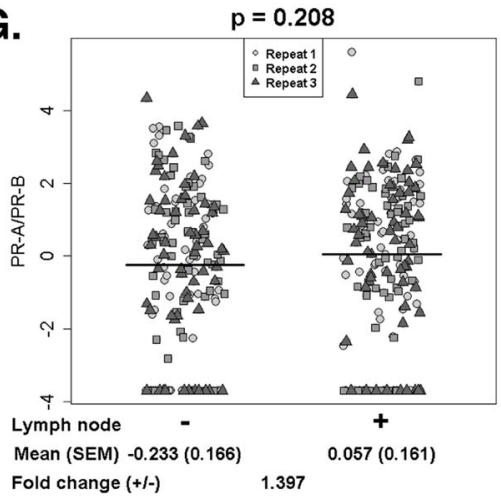

B.

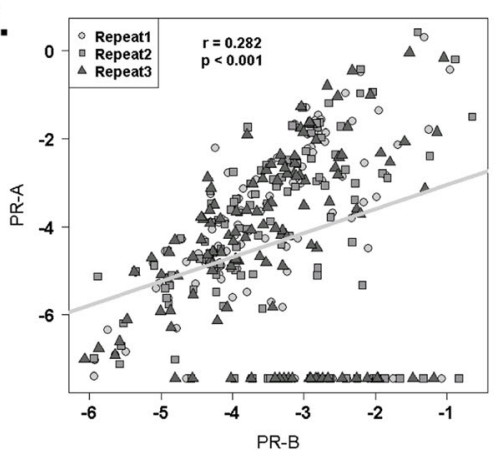

D.

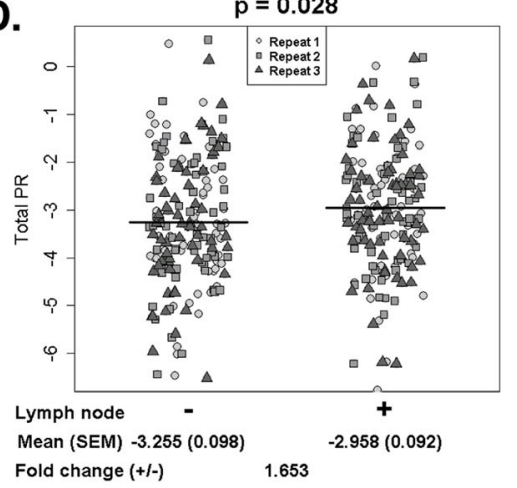

F.

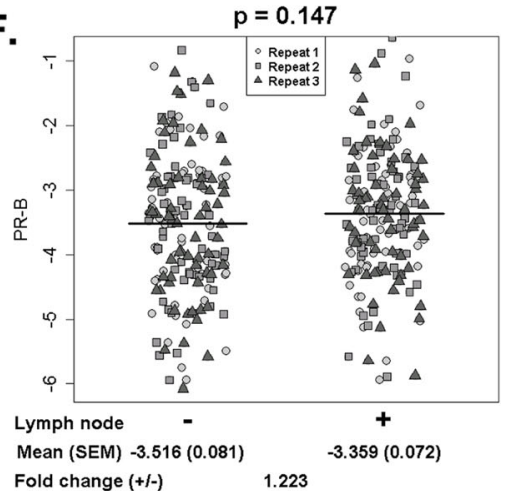

H.

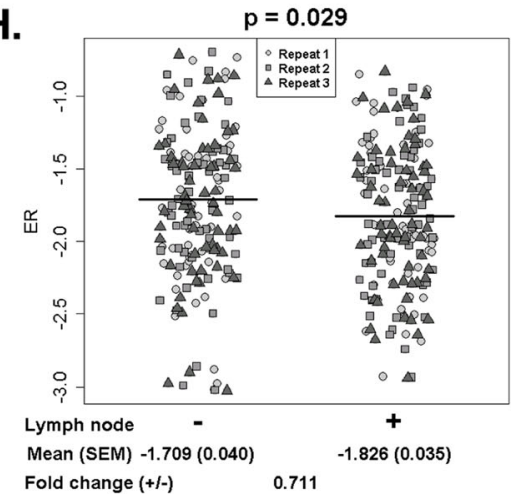

Fig. 2 (See legend on next page.) 
(See figure on previous page.)

Fig. 2 Relationships among total PR, PR-A, PR-B and ER and their associations with lymph node status. Scatter plots of total PR vs. PR-A (Panel a), PR-B vs. PR-A (Panel b), and ER vs. PR-A (Panel c). In Panels A-C, the relative gene expression values are represented after Box-Cox transformation. The solid line represents the linear regression line. The correlation coefficients ( $r$ ) in repeated measures were estimated using linear mixed-effects models. Associations between lymph node status versus total PR (Panel d), PR-A (Panel e), PR-B (Panel f), PR-A/PR-B (Panel g), and ER (Panel h). In Panels D-H, the relative gene expression values are represented after Box-Cox transformation. The solid lines represent the overall mean values. The associations in repeated measures were assessed using a linear mixed-effects model. The transformed expression levels in each group were summarized by mean and standard error of mean (SEM). The fold change (lymph node positive vs. negative) was calculated using the medians of raw expression levels

and in invasive lesions, where a PR-A: PR-B ratio $>1$ was more frequent than a ratio $<1$ [23]. As the extent of dysregulated expression varied from cell to cell within the lesions, the authors suggested that loss of coordinate expression of the PR isoforms is an early event in the development of malignancy with potential implications in tumor progression [23]. Another study conducted using axillary node-positive breast tumors noted that an elevated PR-A: PR-B ratio was more frequently the result of overexpression of PR-A and that patients harboring primary tumors with higher PR-A: PR-B ratios were $\sim 2.8-$ fold more likely to relapse [24]. Our more recent in vitro mechanistic and pre-clinical model studies have rigorously established that PR-A causes invasiveness and metastasis in response to progesterone levels in its pre- and post-menopausal ranges by counteracting the effect of estrogen $[8,9]$. Moreover, elevated expression of PR-A hyper-sensitizes the receptor to progesterone levels at the low end of its post-menopausal range $[8,9]$. We have previously established and reported that in primary $\mathrm{ER}+$ breast cancer tumors, the mRNA levels of PR-A and PR-B correlate with their respective protein levels [9]. The present study directly links this mechanistic role of PR-A with metastasis of L-BCa in a patient population while offering a basis for the previous clinical observations noted above.

The distinctive relationships of the expression levels of PR-A, PR-B and ER in primary L-BCa lesions with node involvement is entirely consistent with predictions based on the previously established mechanistic model (Fig. 1) in which PR-A and PR-B have independent roles in promoting invasiveness and metastasis, with PR-A alone being responsive to the entire range of pre- and postmenopausal progesterone levels, functioning by counteracting the influence of estrogen/ER. As predicted from the model, PR-A was strongly associated with node involvement both in a univariable analysis and after adjusting for age, pathologic type, tumor grade and PR-B. In contrast, PR-B did not show an association with node involvement. ER actually showed a negative correlation with node involvement in the univariable analysis but in the multivariable analysis, this association was weaker, suggesting a more complex role for ER in the pathophysiology of L-BCa. The patients included in this study had predominantly HER2-negative tumors and there was no apparent influence of HER2 on the association of PRA with node involvement, consistent with the previous mechanistic studies.

Together with previous studies, this study supports the view that progesterone, acting largely through PR-A, contributes to metastasis of L-BCa. However, even in post-menopausal women, it would not be feasible to completely inhibit the actions of progesterone to suppress invasion and metastasis of L-BCa. This is because in these women progesterone is needed to check proliferative effects of estrogen on the uterus. In mice in which the PR-A isoform was selectively knocked out, estrogen and even progesterone (acting via PR-B) caused hyperplasia of the uterine epithelium [40]. However, this rodent model data on the physiological role of PR-A in the uterus do not necessarily appear to be able to predict the likely side effects of pharmacologically selectively

Table 2 Associations with lymph node status. The univariable analysis shows the associations between each RNA and lymph node status, while the multivariable analysis presents the associations after adjusting the effects of age, histology, and grade. All the analyses were carried out using linear mixed-effect models. The estimate and SE represent the estimated slope and its associated standard error of lymph node positive

\begin{tabular}{|c|c|c|c|c|c|c|}
\hline & \multicolumn{3}{|c|}{ Univariable analysis } & \multicolumn{3}{|c|}{ Multivariable analysis } \\
\hline & Estimate (SE) & t-value & $\mathrm{p}$ & Estimate (SE) & t-value & $\mathrm{p}$ \\
\hline Total PR & $0.297(0.135)$ & 2.205 & 0.028 & $0.315(0.137)$ & 2.305 & 0.022 \\
\hline PR-B & $0.157(0.108)$ & 1.453 & 0.147 & $0.154(0.110)$ & 1.401 & 0.162 \\
\hline PR-A & $0.553(0.210)$ & 2.626 & 0.009 & $0.639(0.214)$ & 2.994 & 0.003 \\
\hline ER & $-0.116(0.053)$ & -2.194 & 0.029 & $-0.082(0.051)$ & -1.593 & 0.112 \\
\hline PR-A/PR-B & $0.291(0.231)$ & 1.260 & 0.208 & $0.408(0.234)$ & 1.742 & 0.082 \\
\hline
\end{tabular}




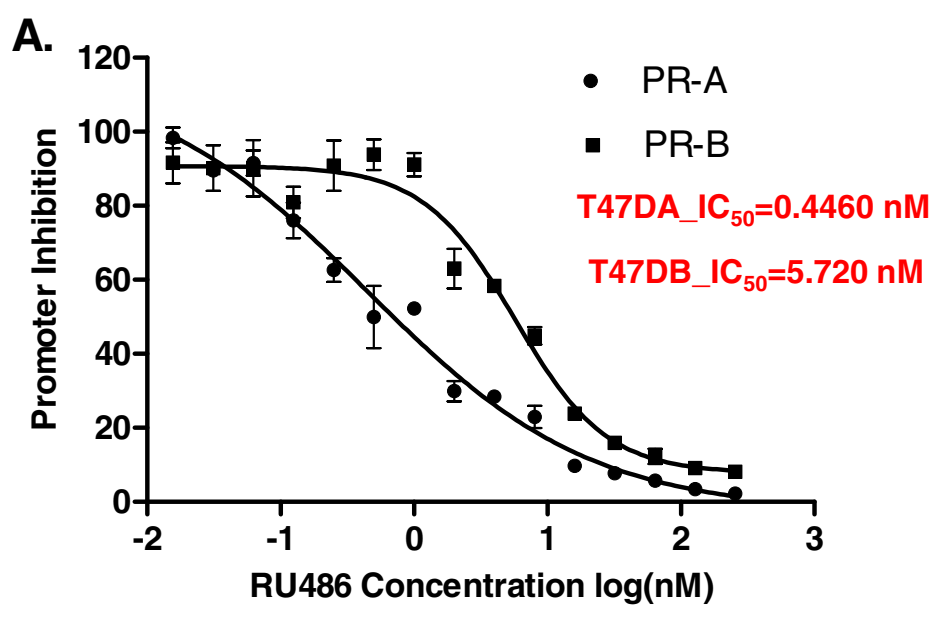

B.

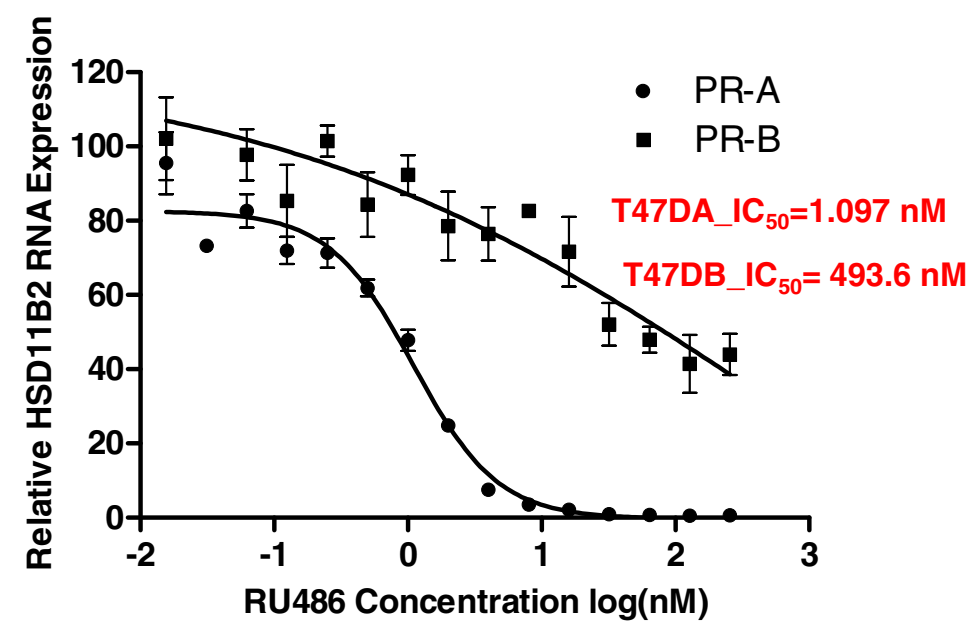

Fig. 3 Mifepristone dose sensitivity for inhibition of the transcriptional activity of PR-A compared with PR-B. In Panels a, hormone depleted T47D$A$ and T47D-B cells were transfected with a plasmid expressing a minimal promoter-luciferase reporter containing a progesterone responsive element (PRE-TATA-LUC) and simultaneously treated with vehicle (DMSO) or $10 \mathrm{nM}$ R5020 (P) plus varying concentrations of RU486 (0.015625 nM$256 \mathrm{nM}$ ) for $48 \mathrm{~h}$. Cells were harvested by preparing lysates for measurement of luciferase activity. In Panel b, T47D-A and T47D-B cells were similarly treated but without transfection. At the end of the treatment, total RNA was isolated and mRNA for the HSD11B2 gene was measured by quantitative RT-PCR. For Panels $\mathbf{a}$ and $\mathbf{b}$, error bars have been included to denote SEM for 3 replicates

modulating PR-A in humans to suppress metastasis of $\mathrm{L}-\mathrm{BCa}$, when the following observations are considered together. First, we noted in this study that the general transcriptional activities of PR-A and PR-B are differentially sensitive to mifepristone, with PR-A being inhibited in a much lower dose range of mifepristone than PR-B. Second, as previously reported by us, a low dose $(1 \mathrm{nM})$ of mifepristone completely inhibited the ability of PR-A to rescue invasiveness of L-BCa cells from suppression by estrogen [8]. In contrast, under the same conditions, mifepristone had no effect on the estrogen-independent induction of invasiveness by PR-B [8]. Finally, in (premenopausal) women treated long-term daily with low dose mifepristone as a potential contraceptive, endometrial hyperplasia was not a significant concern (41). It is therefore possible that in humans, PR-A is selectively targeted by low dose mifepristone, with minimal side effects on the uterus. From a mechanistic standpoint, hormone-dependent transcriptional activities of PR-A and PR-B are quite distinct from each other both in their patterns of regulation of mRNAs [19-21] and micro RNAs [9]. There is a clear structural basis for this functional difference between the PR isoforms, i.e., the amino-terminal 164 residues of PR-B that is lacking in PR-A. Therefore it should be possible in principle to develop selective modulators of PR-A that could potentially offer an effective means of intervention in progression of $\mathrm{L}-\mathrm{BCa}$ in certain patient groups such as post-menopausal women at high risk. 


\section{Conclusion}

Taken together, the current clinical study, our previous detailed mechanistic studies and other related studies in the literature strongly support the view that, throughout a woman's pre- and post-menopausal years, PR-A is a major driver in promoting invasiveness and metastasis of L-BCa by suppressing specific aspects of estrogen/ER action. The studies also suggest that PR-A isoform specific modulation is achievable as a possible intervention for progression of L-BCa in clinically appropriate situations.

\section{Supplementary information}

Supplementary information accompanies this paper at https://doi.org/10. 1186/s12885-020-07002-0.

Additional file 1: Table S1. The univariable analysis shows the association between PR-A and lymph node status and the multivariable analysis shows the associations after adjusting the effects of PR-B along with age, histology, and grade. All the analyses were carried out using linear mixed-effect models. The estimate and SE represent the estimated slope and its associated standard error of lymph node positive. Table S2. The expression levels were dichotomized by the median values for each RNA (Low vs. High). The univariable analysis shows the associations between each RNA and lymph node status, while the multivariable analysis presents the associations after adjusting the effects of age, histology, and grade. All the analyses were carried out using logistic mixed-effect models. OR and Cl stand for 'odds ratio' and 'confidence interval'. Table S3. The expression levels were dichotomized by the median values for each RNA (Low vs. High). The univariable analysis shows the associations between PR-A and lymph node status, while the multivariable analysis presents the associations after adjusting the effects of PR-B along with age, histology, and grade. All the analyses were carried out using logistic mixed-effect models. OR and Cl stand for 'odds ratio' and 'confidence interval'. Table S4. Associations with lymph node status. The univariable analysis shows the associations between each RNA and lymph node status, while the multivariable analysis presents the associations after adjusting the effects of age, histology, grade, and HER2 status. All the analyses were carried out using linear mixed-effect models. The estimate and SE represent the estimated slope and its associated standard error of lymph node positive.

Additional file 2: Figure S1. The distribution of GAPDH Ct values (a) and the associations between GAPDH Ct value and each of lymph node (b), histologic type (c), and grade (d). In (c)-(d), the horizontal bars indicate a mean value and the $p$-value was calculated using repeated measures two-sample t-test and one-way ANOVA under linear mixedeffects models.

\section{Abbreviations}

L-BCa: Luminal breast cancer; PR: Progesterone receptor; PR-A: Progesterone receptor A; PR-B: Progesterone receptor B; ER: Estrogen receptor;

DFS: Disease free survival; DCIS: Ductal carcinoma in situ; miRNA: Micro RNA; TCGA: The Cancer Genome Atlas; CHTN: Cooperative Human Tissue Network; qPCR: Real-time quantitative PCR

\section{Acknowledgements}

Not applicable.

\section{Authors' contributions}

RR planned and conducted most experiments, analyzed data and contributed to the writing of the manuscript. KO planned and conducted experiments. YH provided technical support. SK conducted the statistical analyses and contributed to the writing of the manuscript. MR directed all aspects of the project and also contributed to the writing of the manuscript. All authors have read and approved the manuscript.

\section{Funding}

K.O was supported by a Medical Student Summer Research Fellowship awarded by Wayne State University School of Medicine Medical Student Research Programs. The Biostatistics Core facilities used in this study were supported NIH Center grant P30CA22453 to The Karmanos Cancer Institute. The funding bodies were not involved in in the design of the study and collection, analysis, and interpretation of data and in writing the manuscript.

\section{Availability of data and materials}

Data and plasmid constructs from this study will be made available upon request, following institutional guidelines of Wayne State University.

\section{Ethics approval and consent to participate}

The human tissues were purchased from the Cooperative Human Tissue Network (CHTN), supported by the National Cancer Institute. The patient specimens were collected by CHTN with informed consent and were deidentified by CHTN. This research was exempt with approval from the Wayne State University Institutional Review Board under IRB \#031418MP4X and protocol \#1803001259. The exemption was approved on 03/05/2018 by WSU IRB administration.

\section{Consent for publication}

Not Applicable.

\section{Competing interests}

The authors declare that they have no conflicts of interest.

Received: 3 February 2020 Accepted: 25 May 2020

Published online: 03 June 2020

References

1. Society. AC. Breast cancer facts \& figures 2019-2020. Atlanta: American Cancer Society, Inc; 2019.

2. Siegel RL, Miller KD, Jemal A. Cancer statistics, 2020. CA Cancer J Clin. 2020; 70(1):7-30.

3. Waks AG, Winer EP. Breast cancer treatment: a review. Jama. 2019;321(3): 288-300.

4. Gomez-Fernandez C, Daneshbod Y, Nassiri M, Milikowski C, Alvarez C, Nadji M. Immunohistochemically determined estrogen receptor phenotype remains stable in recurrent and metastatic breast cancer. Am J Clin Pathol. 2008;130(6):879-82.

5. Cardoso F, Harbeck N, Fallowfield L, Kyriakides S, Senkus E, Group EGW. Locally recurrent or metastatic breast cancer: ESMO Clinical Practice Guidelines for diagnosis, treatment and follow-up. Ann Oncol. 2012; 23(suppl_7):vii11-vii9.

6. Zhang XH, Giuliano M, Trivedi MV, Schiff R, Osborne CK. Metastasis dormancy in estrogen receptor-positive breast cancer. Clin Cancer Res. 2013:19(23):6389-97.

7. Kennecke H, Yerushalmi R, Woods R, Cheang MC, Voduc D, Speers CH, et al. Metastatic behavior of breast cancer subtypes. J Clin Oncol. 2010;28(20): 3271-7

8. McFall T, Patki M, Rosati R, Ratnam M. Role of the short isoform of the progesterone receptor in breast cancer cell invasiveness at estrogen and progesterone levels in the pre- and post-menopausal ranges. Oncotarget. 2015:6(32):33146-64

9. McFall T, McKnight B, Rosati R, Kim S, Huang Y, Viola-Villegas N, et al. Progesterone receptor a promotes invasiveness and metastasis of luminal breast cancer by suppressing regulation of critical microRNAs by estrogen. J Biol Chem. 2018:293(4):1163-77.

10. Clemons M, Goss P. Estrogen and the risk of breast cancer. N Engl J Med. 2001;344(4):276-85.

11. Guttilla IK, Adams BD, White BA. ERa, microRNAs, and the epithelialmesenchymal transition in breast cancer. Trends Endocrinol Metab. 2012; 23(2):73-82.

12. Platet N, Cathiard AM, Gleizes M, Garcia M. Estrogens and their receptors in breast cancer progression: a dual role in cancer proliferation and invasion. Crit Rev Oncol Hematol. 2004;51(1):55-67.

13. Sisci D, Maris P, Grazia Cesario M, Anselmo W, Coroniti R, Elvi Trombino G, et al. The estrogen receptor $a$ is the key regulator of the bifunctional role of FoxO3a transcription factor in breast cancer motility and invasiveness. Cell Cycle (Georgetown, Tex). 2013;12(21):3405-20. 
14. Beauchemin D, Lacombe C, Van Themsche C. PAX2 is activated by estradiol in breast cancer cells of the luminal subgroup selectively, to confer a low invasive phenotype. Mol Cancer. 2011;10(1):148.

15. Lin K-H, Wang W-J, Wu Y-H, Cheng S-Y. Activation of antimetastatic Nm23H1 gene expression by estrogen and its a-receptor. Endocrinology. 2002; 143(2):467-75.

16. Rochefort H, Platet N, Hayashido Y, Derocq D, Lucas A, Cunat S, et al. Estrogen receptor mediated inhibition of cancer cell invasion and motility: an overview. J Steroid Biochem Mol Biol. 1998;65(1-6):163-8.

17. Liang Y, Benakanakere I, Besch-Williford C, Hyder RS, Ellersieck MR, Hyder SM. Synthetic progestins induce growth and metastasis of BT-474 human breast cancer xenografts in nude mice. Menopause (New York, NY). 2010; 17(5):1040.

18. Liang Y, Besch-Williford C, Brekken RA, Hyder SM. Progestin-dependent progression of human breast tumor xenografts: a novel model for evaluating antitumor therapeutics. Cancer Res. 2007;67(20):9929-36.

19. Jacobsen $\mathrm{BM}$, Horwitz KB. Progesterone receptors, their isoforms and progesterone regulated transcription. Mol Cell Endocrinol. 2012;357(1-2):1829.

20. Richer JK, Jacobsen BM, Manning NG, Abel MG, Wolf DM, Horwitz KB. Differential gene regulation by the two progesterone receptor isoforms in human breast cancer cells. J Biol Chem. 2002;277(7):5209-18.

21. Shatnawi A, Tran T, Ratnam M. R5020 and RU486 act as progesterone receptor agonists to enhance Sp1/Sp4-dependent gene transcription by an indirect mechanism. Mol Endocrinol (Baltimore, Md). 2007;21(3):635-50.

22. Graham JD, Yager ML, Hill HD, Byth K, O'neill GM, Clarke CL. Altered progesterone receptor isoform expression remodels progestin responsiveness of breast cancer cells. Mol Endocrinol. 2005;19(11):2713-35.

23. Mote P, Bartow S, Tran N, Clarke C. Loss of co-ordinate expression of progesterone receptors $\mathrm{a}$ and $\mathrm{B}$ is an early event in breast carcinogenesis. Breast Cancer Res Treat. 2002;72(2):163-72.

24. Hopp TA, Weiss HL, Hilsenbeck SG, Cui Y, Allred DC, Horwitz KB, et al. Breast cancer patients with progesterone receptor PR-A-rich tumors have poorer disease-free survival rates. Clin Cancer Res. 2004;10(8):2751-60.

25. Barnett JB, Woods MN, Lamon-Fava S, Schaefer EJ, McNamara JR, Spiegelman D, et al. Plasma lipid and lipoprotein levels during the follicular and luteal phases of the menstrual cycle. J Clin Endocrinol Metab. 2004; 89(2):776-82.

26. Johansson ED. Progesterone levels in peripheral plasma during the luteal phase of the normal human menstrual cycle measured by a rapid competitive protein binding technique. Acta Endocrinol. 1969;61(4):592-606.

27. Missmer SA, Eliassen AH, Barbieri RL, Hankinson SE. Endogenous estrogen, androgen, and progesterone concentrations and breast cancer risk among postmenopausal women. J Natl Cancer Inst. 2004;96(24):1856-65.

28. Newton CJ, Samuel DL, James VH. Aromatase activity and concentrations of cortisol, progesterone and testosterone in breast and abdominal adipose tissue. J Steroid Biochem. 1986;24(5):1033-9.

29. Ibrahim YH, Byron SA, Cui X, Lee AV, Yee D. Progesterone receptor-B regulation of insulin-like growth factor-stimulated cell migration in breast cancer cells via insulin receptor substrate-2. Mol Cancer Res. 2008;6(9):14918.

30. Kariagina A, Xie J, Langohr IM, Opreanu RC, Basson MD, Haslam SZ. Progesterone decreases levels of the adhesion protein E-cadherin and promotes invasiveness of steroid receptor positive breast cancers. Horm Cancer. 2013;4(6):371-80

31. Lin VC, Eng AS, Hen NE, Ng EH, Chowdhury SH. Effect of progesterone on the invasive properties and tumor growth of progesterone receptortransfected breast cancer cells MDA-MB-231. Clin Cancer Res. 2001;7(9): 2880-6.

32. Lin VC, Ng EH, Aw SE, Tan MG, Ng EH, Chan VS, et al. Progestins inhibit the growth of MDA-MB-231 cells transfected with progesterone receptor complementary DNA. Clin Cancer Res. 1999;5(2):395-403.

33. Sumida T, Itahana $Y$, Hamakawa H, Desprez P-Y. Reduction of human metastatic breast cancer cell aggressiveness on introduction of either form a or $\mathrm{B}$ of the progesterone receptor and then treatment with progestins. Cancer Res. 2004;64(21):7886-92.

34. Walker NJ. A technique whose time has come. Science. 2002;296(5567):5579.

35. Sakaguchi H, Fujimoto J, Hong BL, Nakagawa Y, Tamaya T. Drastic decrease of progesterone receptor form $B$ but not a mRNA reflects poor patient prognosis in endometrial cancers. Gynecol Oncol. 2004;93(2):394-9.
36. Crowder M. On the use of a working correlation matrix in using generalised linear models for repeated measures. Biometrika. 1995;82(2):407-10.

37. Box G, Cox D. An analysis of transformations. JR Statist Soc E. 1964;26:211-52.

38. Patki M, Chari V, Sivakumaran S, Gonit M, Trumbly R, Ratnam M. The ETS domain transcription factor ELK1 directs a critical component of growth signaling by the androgen receptor in prostate cancer cells. J Biol Chem. 2013;288(16):11047-65.

39. Cianfrocca M, Goldstein LJ. Prognostic and predictive factors in early-stage breast cancer. Oncologist. 2004;9(6):606-16.

40. Mulac-Jericevic B, Mullinax RA, DeMayo FJ, Lydon JP, Conneely OM. Subgroup of reproductive functions of progesterone mediated by progesterone receptor-B isoform. Science. 2000;289(5485):1751-4.

\section{Publisher's Note}

Springer Nature remains neutral with regard to jurisdictional claims in published maps and institutional affiliations.
Ready to submit your research? Choose BMC and benefit from:

- fast, convenient online submission

- thorough peer review by experienced researchers in your field

- rapid publication on acceptance

- support for research data, including large and complex data types

- gold Open Access which fosters wider collaboration and increased citations

- maximum visibility for your research: over $100 \mathrm{M}$ website views per year

At $\mathrm{BMC}$, research is always in progress.

Learn more biomedcentral.com/submissions 